\title{
A frameshift mutation in the LYST gene is responsible for the Aleutian color and associated Chédiak-Higashi syndrome in American mink
}

Anistoroaei, Razvan Marian; Krogh, Anne Kirstine Havnsøe; Christensen, K.

Published in:

Animal Genetics

DOI:

10.1111/j.1365-2052.2012.02391.x

Publication date:

2013

Document version

Early version, also known as pre-print

Citation for published version (APA):

Anistoroaei, R. M., Krogh, A. K. H., \& Christensen, K. (2013). A frameshift mutation in the LYST gene is responsible for the Aleutian color and associated Chédiak-Higashi syndrome in American mink. Animal Genetics, 44(2), 178-183. https://doi.org/10.1111/j.1365-2052.2012.02391.x 


\title{
A frameshift mutation in the LYST gene is responsible for the Aleutian color and the associated Chédiak-Higashi syndrome in American mink
}

\author{
R. Anistoroaei* , A. K. Krogh ${ }^{\dagger}$ and K. Christensen* \\ "Division of Animal Genetics and Bioinformatics, Department of Basic Animal and Veterinary Sciences, The Faculty of Life Sciences, \\ University of Copenhagen, Frederiksberg C, Denmark. ${ }^{\dagger}$ Central Laboratory, Department of Small Animal Clinical Sciences, The Faculty of \\ Life Sciences, University of Copenhagen, 1870, Frederiksberg C, Denmark.
}

\begin{abstract}
Summary
One of the colors of mink is Aleutian (aa)—a specific gun-metal gray pigmentation of the fur-commonly used in combination with other color loci to generate popular colors such as Violet (aаmmpp) and Sapphire (aаpp). The Aleutian color allele is a manifestation of mink Chédiak-Higashi syndrome (CHS), which has been described in humans and several other species. As with forms of CHS in other species, we report that the mink CHS is linked to the lysosomal trafficking regulator (LYST) gene. Furthermore, we have identified a base deletion (c.9468delC) in exon 40 of LYST, which causes a frameshift and virtually terminates the LYST product prematurely (p.Leu3156Phefs*37). We investigated the blood parameters of three wild-type mink and three CHS mink. No difference in the platelet number between the two groups was observed, but an accumulation of platelets between the groups appears different when collagen is used as a coagulant. Microscopic analysis of peripheral blood indicates giant inclusions in the neutrophils of the Aleutian mink types. Molecular findings at the LYST locus enable the development of genetic tests for analyzing the color selection in American mink.
\end{abstract}

Keywords Aleutian mink, Chédiak-Higashi, frameshift, lysosomal trafficking regulator, mutation

\section{Introduction}

Chédiak-Higashi syndrome (CHS) is an autosomal monogenic recessive disorder seen in humans (OMIM \#214500) and other species (cattle, Kunieda et al. 1999; cats, Kramer et al. 1977; killer whales, Ridgway 1979; rats, Nishimura et al. 1989; mice, Lutzner et al. 1967; foxes, Sjaastad et al. 1990; and mink, Padgett et al. 1964, 1967). CHS is expressed by impaired lysosome degranulation with phagosomes, and this induces changes in the nuclear structures of the leukocytes with the accumulation of large lysosomal vesicles in the neutrophils. This can reduce the bactericidal function, leading to susceptibility to some infections. Furthermore, in melanocytes, the melanosomes are not processed normally, resulting in decreased skin

Address for correspondence

R.Anistoroaei, Groennegaardsvej 3, DK- 1870 Frederiksberg C, Denmark.

E-mail: ran@life.ku.dk

Accepted for publication 23 April 2012 pigmentation. Although American mink are not clinically affected by CHS to the same extent as some other species are, the mink model of the disease is interesting from a biological point of view, as hematologic defects and pigment formation and distribution in the fur have the same origin.

In mink, the leukocyte disorder was first found in animals that were homozygous for the Aleutian coat color allele, which results in a silver-grayish gunmetal appearance (Fig. 1a). The Aleutian color is used in combination with other coat color genes to generate many other colors through selective breeding. Currently, only Sapphire (Fig. 1b) and Violet (also known as moylesapphire) (Fig. 1c) are produced on a commercial scale. Although in the past Aleutian mink were reported to be weaker than normal mink and produce smaller litters of weaker kits (Helgebostad 1963), nowadays the mink color types carrying the mutated locus for Aleutian and CHS have a similar production capacity as the standard dark brown mink (wild-type mink) (yearly statistics - Dansk Pelsdyravl, 2010).

Several features of the mink condition partially resemble those of human CHS patients, particularly in relation to the 

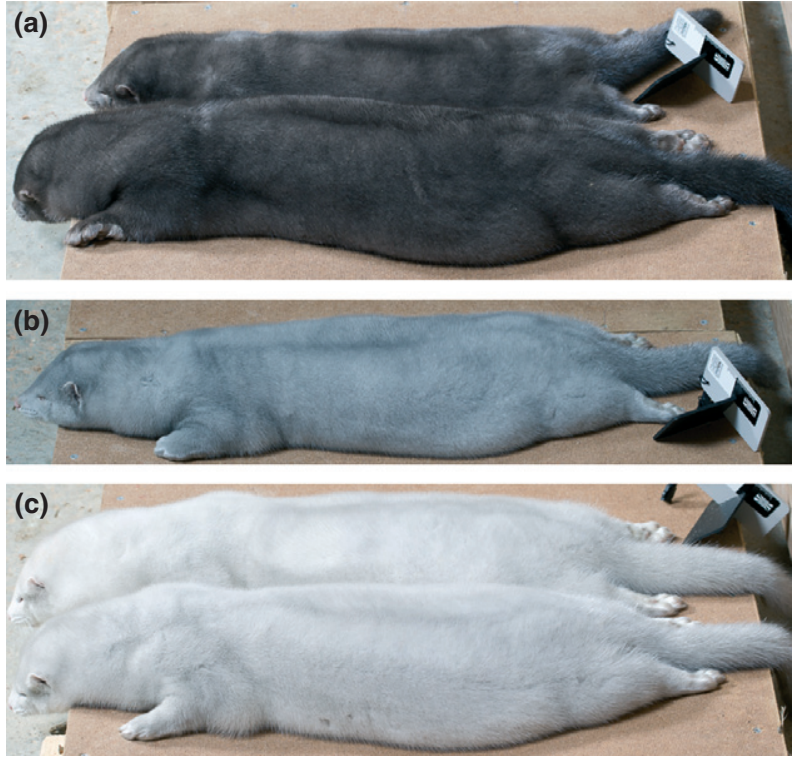

Figure 1 Mink phenotypes homozygous for the LYST mutation. (a) Pure Aleutian (aa); (b) Sapphire (aapp); (c) Violet (aammpp). Larger animals are males and smaller ones are females. Photograph: Jesper Clausen.

blood parameters and diluted pigmentation. Nevertheless, Aleutian mink and mink exhibiting derived colors are not known to display any visible clinical abnormalities (farmers \& vets - personal communication), thus not representing a welfare issue in mink farming.

To date, it has been reported only in humans (OMIM \#214500), beige mice (bg) (Barbosa et al. 1996; Nagle et al. 1996; Perou et al. 1996) and cattle (Kunieda et al. 1999) that CHS is caused by mutations in the LYST gene. This gene encodes a membrane-associated protein, which regulates intracellular protein trafficking. The types and positions of the mutations in the LYST gene vary widely with humans and between species, and they are of interest from a comparative point of view.

In this study, we have identified the LYST gene to be responsible for the mink Aleutian phenotype and the CH-like syndrome. Comparison of LYST gene sequences between wild-type and Aleutian mink revealed a phenotype-specific nucleotide deletion that induces a frameshift and a premature stop of the protein product (p.Leu3156Phefs*37). The typical result of such a mutation may be a complete lack of gene expression and of the LYST protein because the non-sense-mediated decay mechanism
(NMD) would detect the mRNA containing the premature termination codon and degrade it (Strachan \& Read 2010). In addition, examination of blood smears from all the investigated animals indicated giant inclusions in neutrophils correctly segregating with CHS. Supplementary blood analyses of three Aleutian and three wild-type mink also revealed differences in the platelet aggregation in vitro induced by collagen.

\section{Materials and methods}

\section{Family material, markers and genotyping}

A half-sib family originating at the Taastrup Experimental Farm of the Faculty of Life Sciences, University of Copenhagen, Denmark, with 22 offspring that segregated for the Aleutian color (Fig. S1) was genotyped with two microsatellite markers - RAN77 and RAN118 (Table 1) - developed from the assembled contig (JF288176) comprising the candidate LYST gene (Anistoroaei et al. 2011). Genomic DNA was extracted from euthanized mink tongues by using a Promega DNA Extraction Kit (\#A1125). Genotyping was performed with fluorescently labeled forward primers (HEX and NED) on an ABI Prism 3130 sequencer (Applied Biosystems), and genotypes were analyzed using GENSCAN analysis (v.3.1.2) software (Applied Biosystems).

\section{Sequences analysis}

For analyzing of the LYST gene, we initially designed primers on the basis of cDNA and obtained cDNA from CHS mink. Because some of the products could not be amplified and sequenced, genomic DNA was used instead. Thus, primers were designed from the flanking intronic regions of all exons of the LYST gene of the mink (JF288176; Anistoroaei et al. 2011) with PRIMER3 software (http://frodo.wi.mit.edu/cgi-bin/primer3/primer3_www.cgi) (Table S1). After PCR amplification, the DNA products were sequenced and mutations were first identified by comparing a single Aleutian individual with a wild-type mink genotype and with the reference sequence (JF288176). In the second round, analogous PCR products spanning the relevant mutations were amplified from the DNA of an additional two Aleutian (aa), two Sapphire (aapp) and two Violet (aаmmpp) minks along with two wild-type minks. Sequencing was performed using BigDye Terminator Cycle Sequencing chemistry (Applied Biosystems), and extension products

\begin{tabular}{llll}
\hline Primer pair name & Forward and reverse primers $\left(5^{\prime}-3^{\prime}\right)$ & Repeat motif & Alle size \\
\hline RAN77 & F: TGCTTGGTATCAATTTCCAGA & $(\mathrm{CA})_{16}$ & $222-226$ \\
& R: CCCTCTGGGTAGTCCTTGAA & & \\
RAN118 & F: ACTTCCCCACCAAAAGCAG & $(\mathrm{CA})_{12} \ldots(\mathrm{GT})_{12}$ & $241-243$ \\
& R: AAGCCAGATGCTAAAGGCTAGT & & \\
\hline
\end{tabular}

Table 1 Microsatellite markers used for testing LYST as candidates for Aleutian and Aleutian-derived phenotypes in mink. 
were separated on an ABI 3130 Automated Nucleic Acid Analyzer (Applied Biosystems). DNA sequences were processed using LASERGENE (DNA Star, Inc.), and the sequences were compared using the BLAST bl2seq at NCBI.

\section{Hematologic characterization}

Peripheral blood smears were stained with a modified Wright's method and analyzed. Approximately $2 \mathrm{ml}$ of whole blood from three wild-type and three Aleutian carriers (two Sapphire and one Violet) was harvested for in vitro tests using heparin with ADP, heparin with collagen, heparin with ASPI and serotonin without heparin respectively as antagonists. All the individuals were healthy mature females (ca. 12 months of age). Blood was harvested by heart puncture after ketaminol/narcoxyl deep anesthesia. Animals were subsequently euthanized. The analyses were performed within $1 \mathrm{~h}$ from the time of harvesting in a multiplate analysis system (Dynabyte Informationssysteme GmbH). Additionally, a TEG 5000 Analyser (Hemonetics) was used for a thromboelastography test.

\section{Results}

\section{Gene description in mink}

Based on the analysis of the assembled LYST gene genomic contig (JF288176), derived from the CHORI231 American mink BAC library (Anistoroaei et al. 2011), the mink LYST gene is predicted to be represented by 53 exons. The coding region consists of $11403 \mathrm{bp}$, which translates into 3801 amino acids. A BLAST comparison of mink and human LYST sequences revealed nucleotide and deduced amino acid sequence similarities of $88 \%$ and $88 \%$ respectively, whereas those between mink and dog (the closest relative with a published LYST sequence) were $92 \%$ and $93 \%$ respectively. The contig provided $10 \mathrm{di}-$ and tetranucleotide repeated motifs, which could be subsequently developed into microsatellite markers. RAN77 and RAN118 (Table 1) were developed and characterized as markers and were used in this study.

\section{Mapping LYST as the causative gene}

The RAN77 and RAN118 microsatellite markers were located in mink chromosome 2, close to the centromere, following the latest mink linkage map (Anistoroaei et al. subm) upgraded from the Anistoroaei et al. (2009) map. Their physical locations also were confirmed on the basis of sequence homology aided by the Zoo-FISH data (Hameister et al. 1997; Graphodatsky et al. 2000). The genotyping of the two markers for animals within a family segregating for the Aleutian phenotype (Fig. S1) revealed informative polymorphism only for RAN118, which yielded an LOD score value of 3.9 with the Aleutian.

Identification of a nucleotide deletion in the LYST gene

A comparison between the Aleutian and wild-type nucleotide sequences of the LYST exons and flanking regions revealed seven SNPs inconsistently associated with the Aleutian phenotype distributed on exons 4 (two SNPs), 9 (one SNP), 16 (three SNPs) and 40 (one SNP). The comparison also revealed a base deletion at nucleotide position 9468 in the middle of exon 40. This generates a frameshift mutation starting at amino acid position 3156 and results in a premature stop codon after amino acid 3193 (c.9468delC $\rightarrow$ p.Leu3156Phefs*37). The mutation showed a perfect co-segregation with the phenotypes of the seven analyzed Aleutian and Aleutian-derived color mink.

\section{Hematology data}

As other studies have indicated, both in mink and in other species (Padgett et al. 1964; Lutzner et al. 1967; Kaplan et al. 2008), one of the main abnormalities of CHS is neutrophils with giant inclusion bodies. Our analyses are invariably in agreement with this finding (Fig. 2). Additionally, no difference in the number of platelets was noticed between the two test groups comprising three wild-type mink and three Aleutian. An in vitro platelet aggregation test indicated that when collagen was used as antagonist, a great difference was observed between the wild-type group [area under the aggregation curve (AU) between 2836 and 3390] and the Aleutian color types group (AU ranging between 966 and 1790). Aggregation data of whole blood on ASPI, ADP and serotonin did not reveal any significant differences between the two groups of individuals. The TEG (thrombelastograph) test indicated normal and similar blood coagulates, and all the biochemical parameters measured remained within similar ranges. Still, more individuals would be needed for a conclusive statement.

\section{Discussion}

\section{The LYST protein and its functions}

The LYST gene encodes for a lysosomal trafficking regulator protein, which is involved in regulating vesicle's size and trafficking them throughout the cells. Melanosomes are lysosome-like vesicles present in the melanocytes, wherein melanin is synthesized and stored before being transferred to neighboring keratinocytes. Functional studies of different LYST domains and known interacting partners of LYST strongly indicate the role of LYST in determining organelle size by controlling membrane fission events (McVey Ward et al. 2003; Durchfort et al. 2011). The hallmark of CHS is 

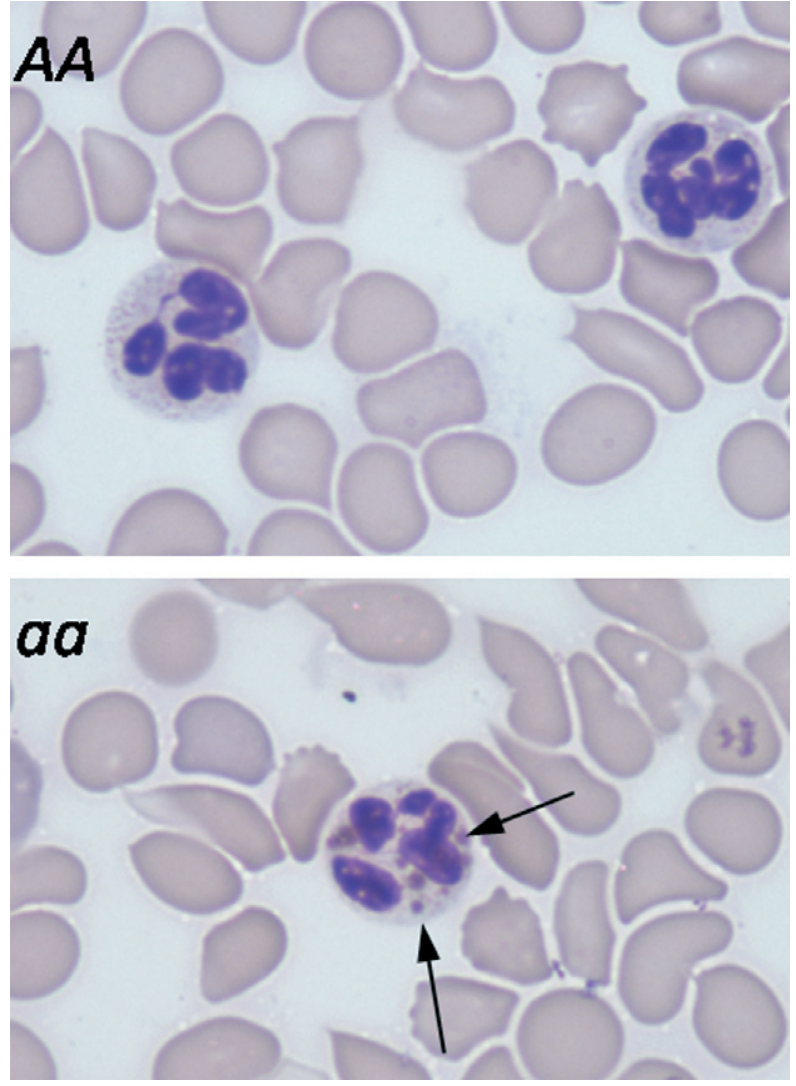

Figure $2 \boldsymbol{A A}$. Normal neutrophils from a peripheral blood smear of wild-type mink; aa. Neutrophils of Aleutian mink displaying giant granules.

giant inclusion bodies in all granulated cells, giant lysosomes and giant melanosomes. Because lysosomes and lysosome-related organelles are involved in waste processing in the cell as well as in pigment production, blood clotting and immune response (Dell'Angelica et al. 2000), their abnormal production would underlie the observed symptoms of CHS. The clinical manifestations of CHS in American mink are almost undetectable in comparison with CHS in humans, who develop a lymphoproliferative syndrome during the so-called accelerated phase. Apart from this difference, the fact that CHS symptoms in mink are less evident than those in humans could also be due to the fact that exposure to infection is limited as the mink farms are normally kept disease free.

\section{Aleutian mink and Aleutian disease infection}

In mink, Aleutian disease (AD) or viral plasmacytosis is a lethal infection, caused by a parvovirus, which results in chronic symptoms of progressive weight loss, lethargy, splenomegaly, anemia, rear leg weakness and seizures. AD was first recognized in farm-raised mink in 1956 and was named as such because it was first identified in mink with the Aleutian coat color. Although it was initially assumed that the disease was a result of poor genetics (and linked to the Aleutian color), it was later found that mink of all coat colors were susceptible to the disease. Importantly, however, mink with other coat colors tended to have lower mortality than did Aleutian mink, as the latter exhibit increased bleeding and a putative immunologic deficiency. Padgett et al. (1967) suggested that $\mathrm{AD}$ is not necessarily linked to the Aleutian genotype and that it may have previously been undiagnosed in other mink color phenotypes. We can presume that the likely explanation for the disease to be initially described and associated with the Aleutian color phenotype is caused by an immune-mediated response to the persistent viral infection that may be dysregulated by the CHS phenotypes owing to the abnormal neutrophils. Therefore, it should be emphasized that there is no exclusive relationship between $\mathrm{AD}$ and the CHS characteristics of Aleutian mink phenotypes.

\section{Availability as an animal model}

Although several species are known to be affected by CHS (Prieur \& Collier 1979), so far only bg mice and rats have been considered as animal models of the disease (Nishimura et al. 1989; Novak et al. 1995). Because mink with CHS (Aleutian and Aleutian-related color phenotypes) are available from many commercial mink ranches, these could also constitute a permanent source of animal models for the syndrome. The approaches may not be directed toward CHS per se but focus upon using cells, platelets and tissues of affected humans and mink to elucidate mechanisms and functions of cells and organelles in the absence of the LYST product (Bell et al. 1976; Buchanan \& Handin 1976; Costa et al. 1976).

\section{The LYST mutation}

Various CHS clinical phenotypes have been associated with different mutations within the LYST gene. The mutations identified in the LYST gene of human CHS patients and of $\mathrm{bg}$ mice (Barbosa et al. 1996, 1997; Nagle et al. 1996; Karim et al. 1997) include various types of frameshift (Table 2) as well as non-sense mutations. Missense mutations in LYST have rarely been reported in humans and mice (Karim et al. 2002; Runkel et al. 2006), but a missense mutation is responsible for CHS in cattle by producing a LYST amino acid substitution (H2015R) (Kunieda et al. 1999). In most of the reported cases, the LYST gene alterations result in a virtually truncated protein. Karim et al. (1997) stated that there is a clear indication that the complete 3801-aminoacid sequence of LYST is required for a CHS-free phenotype in humans. Although RNA expression tests have not been employed in this study, the LYST product is likely to be absent in the CHS animals, as the NMD mechanism is expected to be activated in this case. 
Table 2 Reported frameshift mutations for the LYST gene associated with Chédiak-Higashi syndrome. All mutations were reported in human patients. In bold are the two frameshift mutations in the very proximity of the mutation in American mink.

\begin{tabular}{|c|c|c|}
\hline Type and location & $\begin{array}{l}\text { Premature } \\
\text { termination } \\
\text { codon }\end{array}$ & Reference \\
\hline Single-bp duplication in codon 40 & 50 & $\begin{array}{l}\text { Barbosa et al. (1996); } \\
\text { Nagle et al. (1996) }\end{array}$ \\
\hline Single-bp deletion within codon 489 & 566 & Nagle et al. (1996) \\
\hline Single-bp insertion in codon 633 & 638 & Karim et al. (1997) \\
\hline Single-bp insertion in codon 834 & 898 & Dufourcq-Lagelouse et al. (1999) \\
\hline Single-bp deletion in codon 874 & & Dufourcq-Lagelouse et al. (1999) \\
\hline Two-bp deletion in codon 1024 & 1030 & Barbosa et al. (1997) \\
\hline Single-bp insertion in codon 1028 & 1029 & Barbosa et al. (1997) \\
\hline Single-bp deletion in codon 1772 & 1773 & Certain et al. (2000) \\
\hline Seven-bp deletion in codons $2353-2356$ & 2354 & Certain et al. (2000) \\
\hline Single-bp deletion in codon 2518 & 2519 & Certain et al. (2000) \\
\hline 56-bp deletion in codons 3035-3053 & 3036 & Certain et al. (2000) \\
\hline 10-bp insertion in codon 3076 & 3210 & Certain et al. (2000) \\
\hline Single-bp deletion in codon 3197 & 3258 & $\begin{array}{l}\text { Dufourcq-Lagelouse et al. (1999); } \\
\text { Certain et al. (2000) }\end{array}$ \\
\hline
\end{tabular}

\section{Hematology data}

Blood smear analysis on the Aleutian individuals has been carried out in American mink in previous studies (Padgett et al. 1964; Blume et al. 1969), and our findings are in complete agreement with them. Nevertheless, the TEG and multiplate hematologic investigations performed in this study have never been addressed in this species. Although employed on a small sample size (three wild-type minks and three CHS), there is a clear indication that the platelet aggregation is largely different between the two groups when the whole blood is challenged by collagen.

\section{Conclusion}

In this study, a single-bp deletion within exon 40 (c.9468delC) was identified as being responsible for CHS and the Aleutian and Aleutian loci-derived colors in American mink. The mutation causes a frameshift, which results in a premature stop codon 101 nucleotides downstream. The reported mink mutation disrupts the reading frame from amino acid position 3156 onward, introducing a premature stop codon at position 3195 (p.Leu3156Phefs*37). The identification of this mutation in the LYST gene provides the basis for establishing a DNA selection tool for American minks that are carriers of the recessive Aleutian color phenotype. The condition in mink can serve as potential animal model for addressing CHS caused by a lack of the LYST protein in humans.

\section{Acknowledgements}

This work was supported by Razvan Anistoroaei's postdoctoral grant from the Danish Research Council (\#27820) and funding from the Danish Fur Breeders Association. We are thankful to C. A. Halberg, T. N. Mahler and A. Marrtinsen for the excellent technical assistance in this project and B. Wiinberg for setting TEG working parameters. We acknowledge the excellent help of J. Elnif for the mink blood extraction and B. Pedersen who is in charge of the mink farm.

\section{References}

Anistoroaei R., Ansari S., Farid H., Benkel B., Karlskov-Mortensen P. \& Christensen K. (2009) An extended anchored linkage map and virtual mapping for the American mink genome based on homology to human and dog. Genomics 94, 204-10.

Anistoroaei R., ten Hallers B., Nefedov M., Christensen K. \& de Jong P. (2011) Construction of an American mink bacterial artificial chromosome (BAC) library and sequencing candidate genes important for the fur industry. BMC Genomics 12, 354.

Avlresultater fordelt in farvetyper 2009-2010 (2010) Dansk Pelsdyravl (Journal for Danish Fur breeding) 6, 19 (in Danish). Glostrup: Kopenhagen Fur.

Barbosa M.D.F.S., Nguyen Q.A., Tchernev V.T. et al. (1996) Identification of the homologous beige and Chediak-Higashi syndrome genes. Nature 382, 262-5.

Barbosa M.D.F.S., Barrat F.J., Tchernev V.T. et al. (1997) Identification of mutations in two major mRNA isoforms of the ChediakHigashi syndrome gene in human and mouse. Human Molecular Genetics 6, 1091-8.

Bell T.G., Meyers K.M., Prieur D.J., Fauci A.S., Wolff S.M. \& Padgett G.A. (1976) Decreased nucleotide and serotonin storage associated with defective function in Chediak-Higashi syndrome cattle and human platelets. Blood 48, 175-84.

Blume R.S., Padgett G.A., Wolff S.M. \& Bennett J.M. (1969) Giant neutrophil granules in the Chediak-Higashi syndrome of man, mink, cattle and mice. Canadian Journal of Comparative Medicine 33, 271-4.

Buchanan G.R. \& Handin R.I. (1976) Platelet function in the Chediak-Higashi syndrome. Blood 47, 941-8.

Certain S., Barrat F., Pastural E. et al. (2000) Protein truncation test of LYST reveals heterogenous mutations in patients with Chediak-Higashi syndrome. Blood 95, 979-83. 
Costa J.L., Fauci A.S. \& Wolff S.M. (1976) A platelet abnormality in the Chediak-Higashi syndrome of man. Blood 48, 517-20.

Dell'Angelica E.C., Mullins C., Caplan S. \& Bonifacio J.S. (2000) Lysosome-related organelles. FASEB Journal 14, 1265-78.

Dufourcq-Lagelouse R., Lambert N., Duval M., Viot G., Vilmer E., Fischer A., Prieur M., de Saint Basile G. (1999) Chediak-Higashi syndrome associated with maternal uniparental isodisomy of chromosome 1. European Journal of Human Genetics 7, 633-7.

Durchfort N., Verhoef S., Vaughn M.B., Shrestha R., Adam D., Kaplan J. \& McVey Ward D. (2012) The enlarged lysosomes in beige $_{j}$ cells result from decreased lysosome fission and not increased lysosome fusion. Traffic 13, 108-19.

Graphodatsky A., Yang F., Serdukova N., Perelman P., Zhdanova N. \& Ferguson-Smith M. (2000) Dog chromosome-specific paints reveal evolutionary inter- and intrachromosomal rearrangements in the American mink and human. Cytogenetics and Cell Genetics 90, 275-8.

Hameister H., Klett C., Bruch J., Dixkens C., Vogel W. \& Christensen K. (1997) Zoo-FISH analysis: the American mink (Mustela vison) closely resembles the cat karyotype. Chromosome Research 5, 5-11.

Helgebostad A. (1963) The Aleutian disease. Fur Trade Journal of Canada 40, 10.

Kaplan J., De Domenico I. \& Ward D.M. (2008) Chediak Higashi syndrome. Current Opinion in Hematology 15, 22-9.

Karim M.A., Nagle D.L., Kandil H.H., Burger J., Moore K.J. \& Spritz R.A. (1997) Mutations in the Chediak-Higashi syndrome gene (CHS1) indicate requirement for the complete 3801 amino acid CHS protein. Human Molecular Genetics 6, 1087-9.

Karim M.A., Suzuki K., Fukai K. et al. (2002) Apparent genotypephenotype correlation in childhood, adolescent, and adult Chediak-Higashi syndrome. American Journal of Medical Genetics 108, 16-22.

Kramer J.W., Davis W.C. \& Prieur D.J. (1977) The Chediak-Higashi syndrome of cats. Laboratory Investigation 36, 554-62.

Kunieda T., Nakagiri M., Takami M., Ide H. \& Ogawa H. (1999) Cloning of bovine LYST gene and identification of a missense mutation associated with Chediak-Higashi syndrome of cattle. Mammalian Genome 10, 1146-9.

Lutzner M.A., Lowrie C.T. \& Jordan H.W. (1967) Giant granules in leukocytes of the beige mouse. Journal of Heredity 58, 299-300.

McVey Ward D., Shiflett S.L., Huynh D., Vaughn M.B., Prestwich G. \& Kaplan J. (2003) Use of expression constructs to dissect the functional domains of the CHS/beige protein: identification of multiple phenotypes. Traffic 4, 403-15.

Nagle D.L., Karim M.A., Woolf E.A. et al. (1996) Identification and mutation analysis of the complete gene for Chediak-Higashi syndrome. Nature Genetics 14, 307-11.

Nishimura M., Inoue M., Nakano T., Nishikawa T., Miyamoto M., Kobayashi T., Kitamura Y. (1989) Beige rat: a new animal model of Chediak-Higashi syndrome. Blood 74, 270-3.
Novak E.K., McGarry M.P. \& Swank R.T. (1995) Correction of symptoms of platelet storage pool deficiency in animal models for Chediak-Higashi syndrome and Hermansky-Pudlak syndrome. Blood 66(5), 1196-201.

Padgett G.A., Leader R.W., Gorham J.R. \& O'Mary C.C. (1964) The familial occurrence of the Chediak-Higashi syndrome in mink and cattle. Genetics 49, 505-12.

Padgett G.A., Reiquam C.W., Gorham J.R., Henson J.B. \& O'Mary C. C. (1967) Comparative studies of the Chediak-Higashi syndrome. American Journal of Pathology 51, 553-71.

Perou C.M., Moore K.J., Nagle D.L. et al. (1996) Identification of the murine beige gene by YAC complementation and positional cloning. Nature Genetics 13, 303-8.

Prieur D.J. \& Collier L.L. (1979) Animal model: Chediak-Higashi syndrome of animals. Animal Model of Human Disease 90, 533-6.

Ridgway S.H. (1979) Reported causes of death of captive killer whales (Orcinus orca). Journal of Wildlife Diseases 15, 99-104.

Runkel F., Bussow H., Seburn K.L. et al. (2006) Grey, a novel mutation in the murine Lyst gene, causes the beige phenotype by skipping of exon 25. Mammalian Genome 17, 203-10.

Sjaastad O.V., Blom A.K., Stormorken H. \& Nes N. (1990) Adenine nucleotides, serotonin, and aggregation properties of platelets of blue foxes (Alopex lagopus) with the Chediak-Higashi syndrome. American Journal of Medical Genetics 35, 373-8.

Strachan T. \& Read A. (2010) Human genetic variability and its consequences. Human Molecular Genetics 13, 418.

\section{Supporting information}

Additional supporting information may be found in the online version of this article.

Figure S1 Pedigrees segregating for Aleutian phenotypes in American mink. Circles represent females, squares represent males, solid symbols represent Aleutian color homozygotes (aa) and clear symbols represent wild-type (homo- or heterozygous; $A A$ or $A a$ ) individuals. All parents are heterozygous for the Aleutian with CHS mutation.

Table S1 Primer sequences for LYST exons.

As a service to our authors and readers, this journal provides supporting information supplied by the authors. Such materials are peer-reviewed and may be re-organized for online delivery, but are not copy-edited or typeset. Technical support issues arising from supporting information (other than missing files) should be addressed to the authors. 